\title{
Natural polyphenols improve erectile function and lipid profile in patients suffering from erectile dysfunction
}

\author{
Trebaticky $\mathrm{B}^{1+}$, Muchova $\mathrm{J}^{2+}$, Ziaran $\mathrm{S}^{1}$, Bujdak $\mathrm{P}^{1}$, Breza J, Durackova $\mathrm{Z}^{2}$ \\ Department of Urology, Faculty of Medicine, Comenius University and University Hospital Bratislava, \\ Slovakia. zdenka.durackova@fmed.uniba.sk
}

\begin{abstract}
OBJECTIVES: Erectile dysfunction (ED) is characterised as the inability to achieve or maintain an erection to complete sexual intercourse. ED may be considered as an early complication of diabetes mellitus (DM). The aim of this study was to assess the effect of registered food supplement, natural polyphenolic extract from the French maritime pine bark, Pycnogenol (PYC) on erectile function and lipid profile in ED patients. METHODS: 53 patients with ED were divided into two groups (32 with DM, 21 non-DM) in randomised, blinded and placebo-controlled study. During 3-month intervention with PYC or placebo and one month after the end of the intervention patients were investigated for ED with validated questionnaire International Index of Erectile Function-5 (IIEF-5); lipid profile, glycaemia was analysed in each group.

RESULTS: In a randomised, blinded and placebo-controlled study, we found that natural polyphenolic extract, Pycnogenol improved erectile function in DM group by $45 \%$ compared to the NDM group, where the improvement was also significant, but only by $22 \%$. Total cholesterol, LDL-cholesterol and glucose level was lowered by PYC in patients with DM. Glucose level was not affected by PYC in non-DM. Placebo showed no effect on monitored parameters in both groups.

CONCLUSION: Administration of Pycnogenol leads in improvement of erectile function in patients with ED and diabetes (DM group) by $45 \%$, in NDM group by $22 \%$, in lowering of total-, LDL-cholesterol by $20 \%$ and $21 \%$ and glycaemia by $22 \%$ in DM (Tab. 2, Fig. 2, Ref. 19). Text in PDF www.elis.sk. KEY WORDS: erectile dysfunction, diabetes mellitus, Pycnogenol, total cholesterol, LDL-cholesterol.
\end{abstract}

\section{Introduction}

Erectile dysfunction (ED) is characterised as the inability to achieve or maintain an erection to complete sexual intercourse or another chosen sexual activity sufficiently $(1,2,3,4,5)$. It is known that ED is more common in patients with type 2 diabetes mellitus (DM) and metabolic syndrome (MS) $(6,7)$.

Although ED is present in many non-diabetic men, ED may be considered as an early diabetic complication. However, in case of DM some specific aspects should be taken into account (8): pathogenesis of diabetic ED is specific and more complex compared to non-DM patients; ED is more severe and significantly affects patient lives and the effectiveness of the treatment is lower in DM than in non-DM patients (1).

Pathophysiology of DM and ED has some common risk factors including endothelial dysfunction, MS, insulin resistance

${ }^{1}$ Department of Urology, Faculty of Medicine, Comenius University and University Hospital Bratislava, Slovakia, and ${ }^{2}$ Institute of Medical Chemistry, Biochemistry and Clinical Biochemistry, Faculty of Medicine, Comenius University, Bratislava, Slovakia

+equal contribution

Address for correspondence: $\mathrm{Z}$. Durackova, Ing, $\mathrm{PhD}$, Institute of Medical Chemistry, Biochemistry and Cliical Biochemistry, Faculty of Medicine, Comenius University, Sasinkova 2, SK-813 72 Bratislava, Slovakia. Acknowledgements: This work was supported by VEGA grant of Ministry of Education of SR VEGA 1/0224/08 and Mind and Health, c.a. (Rozum a Zdravie, o.z.). and dyslipidemia (9). When combine with hyperglycaemia, they participate in increased production of pro-inflammatory factors, increased oxidative stress and visceral adiposity which lead to vasoconstriction pathways and decreased $\mathrm{NO}$ availability in corpora cavernosa resulting in ED (10).

In addition to various drugs, many natural substances can interfere with the pathophysiological processes of ED. One well characterised source of polyphenols represents registered food supplement, the French maritime pine (Pinus pinaster) bark standardized extract Pycnogenol (hereafter referred to as PYC). The positive biomodulating effect of polyphenols from the French pine bark has been known since the 16th century, when it was described by the traveler Jacues Cartier in his book Voyages Au Canada. The main constituent of polyphenols in PYC consists of $70 \%$ procyanidins. PYC has significant antioxidant and multiple biomodulating effects such as inhibition of transcription factor $\mathrm{NF}-\kappa \mathrm{B}$, cyclooxygenase, stimulation of NO hypertensive effect by inhibition of angiotensin converting enzyme, antimutagenic effects, alleviation of symptoms of allergic asthma and antiglycaemic effect. In patients with type 2 DM, PYC at a daily dose of $100 \mathrm{mg}$ significantly reduced glucose levels (11). Our previous study shows that PYC improves erectile function and adjusts lipid profile in patients suffering from ED without DM (12).

Trebatický et al (13) suggest that comorbid DM affects the severity of ED. Based on these findings, we performed randomized, placebo-controlled study to assess the PYC administration on erectile function and lipid profile in non-DM and DM patients with ED. 


\section{Subjects and methods}

In a double-blind, randomized, placebo-controlled study, 53 patients (average age $49.0 \pm 11 \mathrm{y}$ ) with ED diagnosed on the basis of the IIEF-5 questionnaire were enrolled. Patients were registered at the Department of Urology of the Faculty of Medicine of Comenius University and University Hospital. The study was approved by the Ethics Committee of the Medical faculty and University Hospital in Bratislava. Patients were enrolled in the study after prior written informed consent.

Study inclusion criteria: diagnostic criteria for ED (IIEF-5 score $<20$ ), normal testosterone levels. All patients enrolled in the study had at least one of the ED risk factors.

Study exclusion criteria: Patients with acute inflammatory diseases, renal failure, hepatic insufficiency, endocrine abnormalities (testosterone deficiency) and psychiatric disorders. No patient with a clear psychogenic ED was included.

Dividing patients into PYC or placebo (PL) groups:

PYC group included 32 patients who received $120 \mathrm{mg}$ PYC daily. PL group included 21 patients received placebo with lactose $(58 \mathrm{mg})$ and cellulose $(65 \mathrm{mg})$ in 1 tablet.

PYC and PL tablets were packaged in the same, opaque package, indicating the name of the clinical study. Patients were on standard diet throughout the 4-month follow-up and did not take any supplementary vitamins with antioxidant properties, vitamin $\mathrm{C}$ and $\mathrm{E}$, and other drugs to treat ED.

PYC group was divided into a subgroup of 17 patients without co-morbid DM (NDM) and a subgroup of 15 patients with ED with DM. A group of ED patients receiving placebo was divided into a subgroup of 11 patients without co-morbid DM (NDM) and a group of 10 ED patients with DM.

Patients were examined at baseline (investigation 0) after one, two and three month of PYC / PL administration (investigation 1, 2,3 ) and one month after discontinuation of intervention (wash-out period, investigation 4). At each stage of the study (visit $0,1,2,3$, 4), patients were examined for clinical state of erectile function and potential adverse events. Erectile function was evaluated by the International Index of Erectile Function (IIEF-5) questionnaire. Patients filled out the IIEF-5 questionnaire during their appointment at the Department of Urology.

Blood for biochemical examinations was collected at $0,1,3$ and 4 visits. Serum was obtained by the standard procedure from blood samples by centrifugation in the absence of anticoagulant. Serum aliquoted samples were kept at $-80{ }^{\circ} \mathrm{C}$ until analyses.

Basic biochemical parameters, glucose, high sensitive C-reactive protein (hsCRP), HbAlc, triacylglycerols (TAG), total cholesterol (TCH), LDL-cholesterol (LDL-CH) and HDL-cholesterol (HDL-CH) in serum were determined at the Department of Laboratory Medicine, University Hospital by standard biochemical procedures using the Hitachi 911 automatic analyser (Roche, Switzerland).

\section{Statistical analysis}

The StatDirect 2.3.7 (StatsDirect Sales, Sale, Cheshire M33 $3 \mathrm{UY}, \mathrm{UK}$ ) was used to randomize and calculate the number of patients enrolled (study strength).
Data are presented as mean $\pm \mathrm{SD}$ or as median (centile 5-95) depending on the normality of data.

Differences in measured parameters between patient groups (PYC and PL) were evaluated by the Student t-test or the MannWhitney $U$ test depending on the normality of data. The effect of treatment was evaluated by Friedman test with Conover comparison of all pairwise.

Statistical analyses were performed with StatsDirect. $\mathrm{p}<0.05$ was considered as significant in all statistical analyses.

\section{Results}

Clinical characteristics and andrology data of patients at the baseline are shown in the Table 1 .

IIEF-5 score, lipid profile and glucose levels at the baseline, at different time of intervention as well as at wash-out period are shown in Table 2.

PYC administered during 3 months significantly improved erectile function in both patient groups. The median value at the baseline is $100 \%$. Patients with diabetes (DM) improved erectile function from the severe ED to moderate ED, an improvement of $45 \%$. Patients without diabetes (NDM) improved erectile function by $22 \%$, but it remains in moderate severity (Fig. 1).

In placebo group, the IIEF-5 score did not change significantly in both groups (DM and NDM) during the intervention (Tab. 2).

Patients reported no adverse effects while PYC and PL administration.

The effect of PYC / PL on the lipid profile and glucose level during 3 months of intervention is shown in Table 2. PYC signifi-

Tab. 1. Clinical characteristics and andrology data of patients at the baseline (investigation 0 ).

\begin{tabular}{|c|c|c|c|c|}
\hline \multirow[b]{3}{*}{ Parameter } & \multicolumn{4}{|c|}{ ED } \\
\hline & \multicolumn{2}{|c|}{ PYC } & \multicolumn{2}{|l|}{ PL } \\
\hline & NDM & $\mathrm{DM}$ & NDM & DM \\
\hline $\mathrm{n}$ & 17 & 15 & 11 & 10 \\
\hline Age (years) & $49.0 \pm 11$ & $48.9 \pm 15$ & $49.9 \pm 12$ & $51.6 \pm 4.4$ \\
\hline Smokers & 10 & 7 & 5 & 4 \\
\hline Arterial hypertension & 7 & 8 & 3 & 4 \\
\hline Ischemic heart disease & 3 & 4 & 2 & 4 \\
\hline Stress & 5 & 7 & 4 & 4 \\
\hline Time since ED diagnosis (months) & $12(3-48)$ & $27(4-57)^{\#}$ & $10(2-35)$ & $25(5-45)^{\#}$ \\
\hline DM treatment - PAD & - & 11 & - & 6 \\
\hline - insulin & - & 2 & - & 0 \\
\hline - antidiabetic diet & - & 2 & - & 2 \\
\hline IIEF-5 in median (Centile 5-95) & $11(8-20)^{\#}$ & $10(5-12)^{* \#}$ & $9(8-15)^{\#}$ & $10(5-9)^{\#}$ \\
\hline severe ED (IIEF-5, 0-10) (\%) & $8(47)$ & $8(53)$ & $5(46)$ & $5(50)$ \\
\hline moderate ED (IIEF-5, 11-15) $(\%)$ & $6(35)$ & $5(34)$ & $4(36)$ & $3(30)$ \\
\hline mild ED (IIEF-5, 16-20) (\%) & $3(18)$ & $2(13)$ & $2(18)$ & $2(20)$ \\
\hline no ED (IIEF -5, 21-25) (\%) & - & - & & \\
\hline $\mathrm{BMI}\left(\mathrm{kg} / \mathrm{m}^{2}\right)$ & $28.3 \pm 2.9$ & $31.9 \pm 1.0^{\#}$ & $27.9 \pm 2.5$ & $30.2 \pm 5.4$ \\
\hline HbA1c (\%) & $3.96 \pm 0.34$ & $7.58 \pm 2.05^{\#}$ & $3.85 \pm 0.33$ & $7.80 \pm 0.42^{\#}$ \\
\hline hsCRP (mg/L) & $4.16 \pm 0.67^{\#}$ & $4.70 \pm 0.70^{\#}$ & $4.21 \pm 0.56$ & $4.84 \pm 0.98^{\#}$ \\
\hline
\end{tabular}

ED - erectile dysfunction, PYC - pycnogenol $\AA$, PL - placebo, NDM - patients without diabetes mellitus, $\mathrm{DM}$ - patients with diabetes mellitus, $\mathrm{PAD}$ - peroral antidiabetics, IIEF-5 - international index of erectile function, BMI - body mass index, $\mathrm{HbA} 1 \mathrm{c}$ - glycated hemoglobin

\#p DM vs NDM 
Table 2. IIEF-5 score, lipid profile and glucose levels at the baseline (investigation 0) and after the intervention and at the wash-out period

\begin{tabular}{|c|c|c|c|c|c|}
\hline \multirow[b]{3}{*}{ Parameter } & \multirow[b]{3}{*}{ Month } & \multicolumn{4}{|c|}{ ED } \\
\hline & & \multicolumn{2}{|c|}{ PYC } & \multicolumn{2}{|c|}{ PL } \\
\hline & & NDM & DM & NDM & DM \\
\hline \multirow[t]{5}{*}{ IIEF-5 } & 0 & 11.6 & $9.0^{\#}$ & 10.8 & 9.7 \\
\hline & 1 & $12.8^{*+}$ & $11.2^{*+}$ & 9.6 & 9.7 \\
\hline & 2 & $12.5^{*+}$ & $11.4^{*+}$ & 7.9 & 8.8 \\
\hline & 3 & $14.1^{*+}$ & $13.1^{*+}$ & 9.5 & 7.8 \\
\hline & 4 & $13.6^{*+}$ & $10.7^{*+}$ & 9.3 & 8.2 \\
\hline \multirow{4}{*}{$\begin{array}{l}\mathrm{TCH} \\
(\mathrm{mmol} / \mathrm{L})\end{array}$} & 0 & $5.46 \pm 0.60$ & $5.70 \pm 0.54$ & $4.73 \pm 1.10$ & $5.29 \pm 0.58$ \\
\hline & 1 & $5.04 \pm 0.66^{*}$ & $4.94 \pm 0,57^{*}$ & $4.73 \pm 1.22$ & $5.41 \pm 0.38$ \\
\hline & 3 & $4.97 \pm 0.63 *$ & $4.55 \pm 0.57^{*}$ & $4.89 \pm 1.34$ & $5.34 \pm 0.42$ \\
\hline & 4 & $5.55 \pm 0.64$ & $4.87 \pm 0.53$ & $4.78 \pm 1.12$ & $5.09 \pm 0.64$ \\
\hline \multirow{4}{*}{$\begin{array}{l}\text { LDL-CH } \\
(\mathrm{mmol} / \mathrm{L})\end{array}$} & 0 & $3.75 \pm 0.51$ & $3.73 \pm 0.37$ & $2.86 \pm 0.85$ & $3.25 \pm 0.49$ \\
\hline & 1 & $3.32 \pm 0.21 *$ & $3.07 \pm 0.52 *$ & $3.01 \pm 1.22$ & $3.27 \pm 0.27$ \\
\hline & 3 & $3.22 \pm 0.63^{*}$ & $2.97 \pm 0.63^{*}$ & $2.74 \pm 1.03$ & $3.28 \pm 0.5$ \\
\hline & 4 & $3.61 \pm 0.69$ & $2.73 \pm 0.73$ & $2.74 \pm 0.92$ & $3.25 \pm 0.83$ \\
\hline \multirow{4}{*}{$\begin{array}{l}\mathrm{HDL}-\mathrm{CH} \\
(\mathrm{mmol} / \mathrm{L})\end{array}$} & 0 & $1.44 \pm 0.31$ & $1.26 \pm 0.13$ & $1.09 \pm 0.28$ & $1.42 \pm 0.40$ \\
\hline & 1 & $1.41 \pm 0.82$ & $1.14 \pm 0.17^{\#}$ & $1.13 \pm 0.33$ & $1.25 \pm 0.5$ \\
\hline & 3 & $1.53 \pm 0.38$ & $1.25 \pm 0.21$ & $1.21 \pm 0.33$ & $1.07 \pm 0.19$ \\
\hline & 4 & $1.48 \pm 0.35$ & $1.31 \pm 0.43$ & $1.23 \pm 0.33$ & $1.08 \pm 0.31$ \\
\hline \multirow{4}{*}{$\begin{array}{l}\text { TAG } \\
(\mathrm{mmol} / \mathrm{L})\end{array}$} & 0 & $1.53 \pm 0.77$ & $1.91 \pm 0 . .77$ & $1.86 \pm 0.61$ & $3.69 \pm 1.50$ \\
\hline & 1 & $1.41 \pm 0.82$ & $2.15 \pm 1.28$ & $1.77 \pm 0.95$ & $4.53 \pm 1.8$ \\
\hline & 3 & $1.25 \pm 0.66$ & $2.21 \pm 0.88$ & $1.87 \pm 0.88$ & $2.38 \pm 1.1$ \\
\hline & 4 & $1.33 \pm 0.64$ & $1.69 \pm 1.03$ & $1.75 \pm 0.95$ & $3.79 \pm 1.45$ \\
\hline \multirow{4}{*}{$\begin{array}{l}\text { Glucose } \\
(\mathrm{mmol} / \mathrm{L})\end{array}$} & 0 & $5.23 \pm 0.39$ & $11.06 \pm 2.3^{\#}$ & $5.28 \pm 0.48$ & $10.08 \pm 1.42^{\#}$ \\
\hline & 1 & $5.14 \pm 0.48^{*}$ & $8.88 \pm 3.15^{* \#}$ & $5.56 \pm 0.41$ & $10.90 \pm 1.71$ \\
\hline & 3 & $5.10 \pm 0.49 *$ & $8.60 \pm 3.27$ *\# & $5.49 \pm 0.74$ & $10.10 \pm 1.36$ \\
\hline & 4 & $5.10 \pm 0.51$ & $8.55 \pm 2.6^{* \#}$ & $5.73 \pm 0.53$ & $9.95 \pm 1.36$ \\
\hline
\end{tabular}

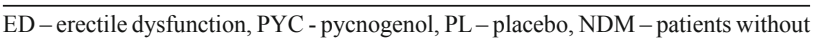
diabetes mellitus, DM - patients with diabetes mellitus, IIEF-5 - international index of erectile function, TCH - total cholesterol, LDL-CH - low density lipoprotein cholesterol, HDL-CH - high density lipoprotein cholesterol, TAG - triacylglycerol, * p W vs W0, ${ }^{*}$ p DM vs NDM, + p PYC vs PL

cantly reduced total cholesterol and LDL-cholesterol, unlike to placebo, regardless of co-morbid DM. HDL-cholesterol and TAG serum levels in DM and NDM subgroup were not significantly affected neither by PYC nor PL administration (Tab. 2).

The difference in the effect of PYC on TCH and LDL-CH levels in DM and NDM is shown in Figure 2. Patients with DM were more sensitive to the effect of PYC on TCH and LDL-CH. After 3 months of PYC administration, TCH in the DM subgroup decreased by $20 \%$ and LDL-CH by $21 \%$ compared to the baseline. In the NDM group, the effect of PYC was significant but less effective compared to diabetics. TCH decreased by $9 \%$ and LDL-CH by $14 \%$ (Fig. 2).

Glucose serum level at the baseline was higher in DM patients compared to NDM patients in both, PYC and PL groups, as expected $(\mathrm{p}<0.0001)$. In DM subgroup, PYC administration significantly decreased glycaemia after one or three months by $20 \%$ and $22 \%$, resp. in contrary to NDM group, where positive effect of PYC was also observed, but without statistical significance.

We evaluated the effect of PYC treatment by the Friedman test. Significant effect was found in both DM and NDM subgroups to improve erectile function (for both subgroups $\mathrm{p}<0.001$ ), to reduce $\mathrm{TCH}$ (for both groups $\mathrm{p}<0.001)$ and LDL-CH $(\mathrm{p}=0.0005, \mathrm{p}=$ $0.035)$. PYC reduced the serum glycaemia significantly only in the diabetic subgroup $(p=0.0001)$ but not in the NDM subgroup

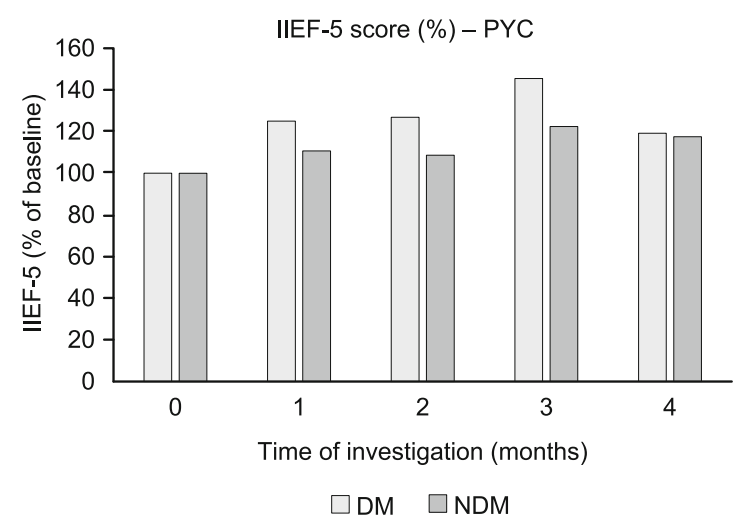

Fig. 1. IIEF-5 score (median) (\%) in DM and NDM groups during intervention of PYC. DM - patients with diabetes, NDM - patients without diabetes, IIEF-5 - International Index of Erectile Function,PYC -pycnogenol.
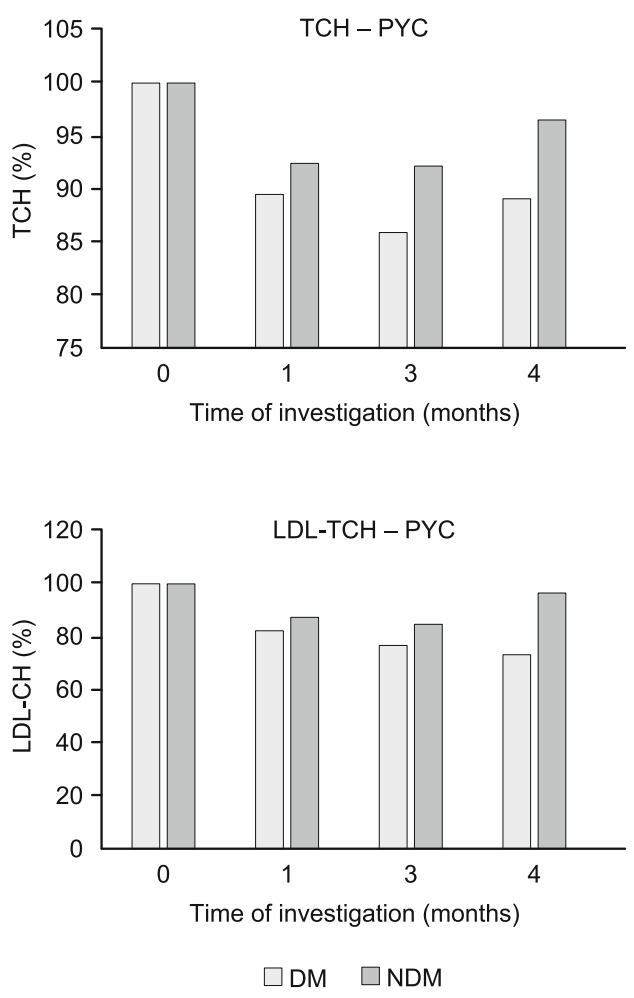

Fig. 2. Total cholesterol and LDL-cholesterol in DM and NDM groups using PYC (\%). TCH - total cholesterol, LDL-CH - low density lipoprotein cholesterol, DM - patients with diabetes, NDM - patients without diabetes, PYC - Pycnogenol.

$(p=0.598)$. The PL administration showed no statistical significance in any subgroup.

\section{Discussion}

Recent studies confirmed that DM significantly contributes to the pathophysiology and manifestation of ED (13). ED is present in many non-diabetic men (NDM), but ED may appear before DM 
941-944

is diagnosed, as both diagnoses share several common features, including endothelial dysfunction (1).

DM is one of the leading causes of cardiovascular disease associated with plethora of health problems lowering quality of life, including sexual dysfunction. DM and ED share several cardiometabolic risk factors, such as endothelial dysfunction, obesity, MS and hormonal inadequacy and dyslipidemia (14). These factors, together with hyperglycemia, participate in endothelial activation, pro-inflammatory state and oxidative stress (15). Increased vasoconstriction and reduced NO availability in corpora cavernosa results in limited erectile function (10).

In ED patients, the erectile function is examined by the IIEF5 self-assessment questionnaire. The maximum score of IIEF-5 questionnaire is 25 points (16). Erectile function is characterised by the following score: normal function (21-25 points), mild erectile dysfunction (16-20 points), moderate erectile dysfunction (11-15 points), severe dysfunction (less than 10 points).

PYC is known to significantly improve moderate ED in men without DM at a daily dose of $120 \mathrm{mg}$ (12) and significantly reduces TCH and LDL-CH $(12,17)$. However, to our knowledge, there are no studies that compare the effect of PYC on ED patients without other major comorbidities and with DM.

Glycaemia lowering effect of PYC is known in rats with streptozotocin-induced diabetes (18) and the antidiabetic effect of a daily dose of PYC $100 \mathrm{mg}$ in patients with DM type 2 (19). Therefore, we conducted study to assess the effect of PYC on erectile function and lipid profile of patients with ED with and without DM. Our results indicate that patients with ED and DM are more sensitive to the positive effect of PYC compared to NDM ED patients. In our recent work (8), we found elevated marker of endothelial dysfunction, endoglin in our patients with DM compared to NDM patients at the baseline which positively correlated with glucose level. PYC decreased glucose levels in DM patients and decreased glucose levels correlated with decreased endoglin. From the correlations found in our previous work (13) follows that decreased endoglin is associated with improved endothelial function, increased NO production and higher IIEF-5 scores (13). Glucose lowering effect of PYC appears to be one of the mechanisms responsible for improving erectile function in diabetic patients with erectile dysfunction.

\section{Conclusion}

Patients suffering from ED and DM type 2 had worse erectile function compared to NDM ED patients. In a randomised, blinded and placebo-controlled study, we found that registered food supplement, natural polyphenolic extract, Pycnogenol (PYC) improved erectile function in DM group by $45 \%$ compared to the NDM group, where the improvement was also significant, but only by $22 \%$.

Administration of PYC in DM patients with ED led to statistically significant reduced glycaemia by $22 \%$ and to lowering of total-, LDL-cholesterol by $20 \%$ and $21 \%$.

PYC seems to have a beneficial effect on treatment of ED especially in patients with DM. This needs to be verified by larger placebo-controlled studies and longer treatment endpoints.

\section{References}

1. Kamenov ZA. A comprehensive review of erectile dysfunction in men with diabetes. Exp Clin Endocrinol Diabetes 2015; 123: 141-158.

2. Laumann EO, Waite LJ. Sexual dysfunction among older adults: prevalence and risk factors from a nationally representative U.S. probability sample of men and women 57-85 years of age. J Sex Med 2008; 5: 2300-2311.

3. Breza J. Diagnosis and treatment of erectile dysfunction. Bratisl Lek Listy 1993; 96 (12): 642-650.

4. Štefančík J, Novotný V, Trebatický B., Breza J. Modern methods of oral treatment of erectile dysfunction. Bratisl Lek Listy 2003; 104 (4-5): 162.

5. Štefančík J, Trebatický B, Breza J. Erectile dysfunction as a symptom of vascular disease. Bratisl Lek Listy 2005; 106 (11): 380.

6. Esposito K, Giugliano F, Martedì E, Feola G, Marfella R, D'Armiento M, Giugliano D. High proportions of erectile dysfunction in men with the metabolic syndrome. Diabetes Care 2005; 28: 1201-1203.

7. Fillo J, Breza J Jr, Ondrušová M, Luha J, Ondruš D, Dúbravický J, Breza J, Labaš P, Levčíková $M$. Results of long term testostertone replacement therapy in men with abdominal obesity, erectile dysfunction and testosterone deficiency. Bratisl lek Listy 2018; 119 (9): 577-580. doi: 10.4149/ BLL_2018_061.

8. Jakuš V, Rietbrock N. Advanced glycation end-products and the progress of diabetic vascular complications. Physiol Res 2004; 53: 131-142.

9. Breza J Jr, Ďuračková Z, Payer J, Dukát A, Breza J Sr. Lipoprotein particle size in patients undergoil kidney transplantation. Bratisl lek Listy 2018; 119 (3): 130-132. doi: 10.4149/BLL_2018_025.

10. Maiorino MI, Bellastella G, Giugliano D, Esposito K. From inflammation to sexual dysfunctions: a journey through diabetes, obesity, and metabolic syndrome. J Endocrinol Invest 2018; 41 (11): 1249-1258.

11. Rohdewald P. Encyclopedia of dietary supplements. Marcel Dekker, 2005. doi:10.1081/E-EDS-120022123.

12. Ďuračková Z, Trebatický B, Novotný V, Žitňanová I, Breza J. Lipid metabolism and erectile function improvement by Pycnogenol, extract from the bark of Pinus pinaster in patients suffering from erectile dysfunction-a pilot study. Nutr Res 2003; 23: 1189-1198.

13. Trebatický B, Žitňanová I, Dvořáková M, Országhová Z, Paduchová Z, Ďuračková Z, Breza J, Muchová J. Role of oxidative stress, adiponectin and endoglin in the pathophysiology of erectile dysfunction in diabetic and non-diabetic men. Physiol Res 2019; 68: 623-631.

14. Melman A, Rehman J. Pathophysiology of erectile dysfunction. Mol Urol 1999; 3: 87-102.

15. Maiorino MI, Bellastella G, Esposito K. Diabetes and sexual dysfunction: current perspectives. Diabetes Metab Syndr Obes 2014; 7: 95-105.

16. Rhoden EL, Telöken C, Sogari PR, Vargas Souto CA. The use of the simplified International Index of Erectile Function (IIEF-5) as a diagnostic tool to study the prevalence of erectile dysfunction. Int J Impot Res 2002; 14: $245-250$.

17. Devaray S, Kaul N, Schönlau F, Rohdewald P, Jialal I. Supplementation with a pine bark extract rich in polyphenols increases plasma antioxidant capacity and alters plasma lipoprotein profile. Lipids 2002; 37 (10): 931-934.

18. Maritim A, Dene BA, Sanders RA, Watkins JB. Effect of Pycnogenol treatment on oxidative stress in streptozotocin-induced diabetic rats. J Biochem Mol Toxicol 2003; 17: 193-199.

19. Liu X, Wei J, Tan F, Zhou S, Wurthwein G, Rohdewald P. Antidiabetic effect of PycnogenolFrench maritime pine bark extract in patients with diabetes type II. Life Sci 2004; 75 (21): 2505-2513.

Received June 26, 2019. Accepted September 19, 2019. 